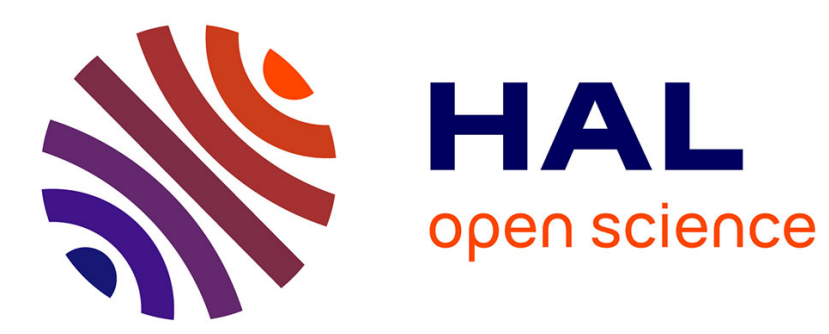

\title{
La prévision hydro-météorologique à 3-6 mois. Etat des connaissances et applications
}

\author{
Eric Sauquet, J. Lerat, C. Prudhomme
}

\section{To cite this version:}

Eric Sauquet, J. Lerat, C. Prudhomme. La prévision hydro-météorologique à 3-6 mois. Etat des connaissances et applications. La Houille Blanche - Revue internationale de l'eau, 2008, 6, p. 77 - p. 84. 10.1051/lhb:2008075. hal-00454536

\section{HAL Id: hal-00454536 https://hal.science/hal-00454536}

Submitted on 8 Feb 2010

HAL is a multi-disciplinary open access archive for the deposit and dissemination of scientific research documents, whether they are published or not. The documents may come from teaching and research institutions in France or abroad, or from public or private research centers.
L'archive ouverte pluridisciplinaire HAL, est destinée au dépôt et à la diffusion de documents scientifiques de niveau recherche, publiés ou non, émanant des établissements d'enseignement et de recherche français ou étrangers, des laboratoires publics ou privés. 


\title{
La prévision hydro-météorologique à 3-6 mois. Etat des connaissances et applications
}

\author{
Seasonal forecasting of river flows - State-of-the-art and applications \\ ERIC SAUQUET \\ Cemagref, Unité de Recherche Hydrologie-Hydraulique \\ 3 bis quai Chauveau, CP 200, 69336 Lyon cedex 09, France \\ Tél : +33 (0)4 722089 13, Fax : +33 (0)4 784778 75, e-mail : eric.sauquet@cemagref.fr
}

\section{JULIEN LERAT}

Cemagref, Unité de Recherche Hydrosystèmes et Bioprocédés

Parc de Tourvoie, BP 44, 92163 Antony cedex, France

Tél : +33 (0)1 409665 52, Fax : +33 (0)1 409661 99, e-mail : julien.lerat@cemagref.fr

\section{CHRISTEL PRUDHOMME}

Centre for Ecology and Hydrology

Maclean Building, Crowmarsh Gifford, Wallingford, Oxfordshire OX10 8BB, United Kingdom Tél : +44 (0)1491 692381, Fax : +44 (0)1491692424, e-mail : c.prudhomme@ceh.ac.uk

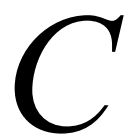

ette étude est centrée sur les méthodes et outils pour la prévision saisonnière des débits. Après un bref état de l'art sur le sujet, nous présentons deux méthodes différentes appliquées aux étiages sur des cours d'eau français. La première approche testée ambitionne de prédire les probabilités d'apparition d'étiages sévères sur les régions hydrologiquement homogènes selon le type de temps du jour. La qualité de la prévision en conditions parfaitement connues montre que la France n'offre pas un cadre favorable d'application. Le deuxième cas d'étude concerne le bassin du Rhin. Le modèle pluie-débit GR4J, le module neige du modèle suédois HBV et un générateur stochastique de pluie ont été assemblés pour restituer les débits journaliers sur 90 jours pour sept stations du bassin du Rhin. Les résultats montrent qu'une prévision fiable à 30 jours est possible à l'aval du bassin, en revanche, l'outil ne parvient pas à anticiper les étiages en amont du bassin (forte réactivité).

$T$

his study focuses on the methodology and tools used in seasonal forecasting of river flows. After a brief state of the art, two applications for predicting low flows of French rivers are presented. The first one aims at forecasting the probability of occurrence of severe droughts using weather typing within hydrologically homogeneous regions. The relatively poor quality of results in validation suggests that France may not be well suited for this type of approach. The second approach is applied to the Rhine River. The rainfall-runoff model GR4J combined with the HBV snow module is forced by a stochastic rainfall generator to provide daily river flows on seven sub-basins for the following 90 days. Results show that a reliable mid term forecasting ( $<30$ days) is possible in the lower catchment but the tool is not able to anticipate low flows in the upper catchment.

\section{INTRODUCTION}

En parallèle d'une gestion efficace de la demande, l'anticipation des pénuries de ressources en eau facilite la tâche du décideur en apportant le délai nécessaire à l'action et à la concertation. Les moyens actuels en matière de calcul numérique et d'accès aux données environnementales permettent d'envisager des prévisions hydro-météorologiques à moyen terme avec des horizons de l'ordre de quelques mois. Le travail ultérieur consiste ensuite à analyser ces données qualifiant l'état des ressources pour les mois à venir et à proposer des règles de gestion adaptées. Dans ce domaine, la prévision de variables météorologiques (pluie, température) est la plus avancée et la plus ancienne avec des travaux datant des années 1950 [1]. Concernant les écoulements en rivière, les recherches se sont concentrées sur la prévision des crues à brève échéance.

Le présent document est une contribution au développement de méthodes de prévision à longue échéance centrées sur les périodes d'étiage pendant lesquelles la gestion de la demande est la plus critique. De telles prévisions représentent un défi scientifique important puisqu'elles réclament : 
- une connaissance régionale voire globale des forçages atmosphériques. Ces forçages conditionnent en effet fortement l'évolution des débits pour les mois à venir ;

- une connaissance locale de l'hydrologie permettant de transformer ces forçages en débits ;

- l'assemblage des deux approches dans un contexte probabiliste compte tenu des incertitudes liées à l'évolution de l'atmosphère. Ainsi les prévisions sont souvent assorties d'un indice de confiance qualifiant la dispersion des valeurs prévues : plus les outils de prévision convergent vers une valeur unique, plus la prévision apparaît comme solide.

Seule la partie naturelle de la ressource en eau est étudiée. Les variations de débit dues à une variation de la demande ne sont pas considérées. Pour traiter cette question, deux grandes approches ont été retenues par les scientifiques. Nous en présentons les principales caractéristiques dans le paragraphe suivant. Deux exemples illustrent nos propos dans les sections II et III. Enfin des pistes d'amélioration sont suggérées en conclusion.

\section{I.1 APPROCHE STATISTIQUE}

L'objectif est d'établir un lien direct entre l'état de l'atmosphère ou de l'océan à l'instant de la prévision et l'occurrence d'événements à l'échéance de quelques mois ou semaines. Il n'est pas recherché de structure interne permettant d'expliquer ce lien. L'avantage d'une telle approche est d'englober en une seule étape l'ensemble des trois éléments décrits précédemment. Il s'agit principalement de rapprocher deux jeux de variables : le premier regroupe les variables explicatives et le second les variables cibles ou des statistiques les renseignant. La difficulté est de déceler des facteurs explicatifs ayant un fondement physique significatif. Ce type d'approches a été appliqué avec succès sur les régions subissant les effets de El Nino [2][3][4]. Plus proche de nous, les travaux sur les débits estivaux de cours d'eau britanniques [5][6] sont certainement les plus avancés. En France, aucune étude n'a à ce jour identifié de " prédicteurs " solides et puissants alimentant ces méthodes.

La variété des méthodes statistiques et la liberté dans le choix des variables d'entrée et de sortie rendent difficile la comparaison entre expériences. Il n'existe pas de consensus clair sur le choix d'un type d'outil statistique. Ces critiques peuvent être également perçues comme des avantages, la souplesse de ces méthodes offre de multiples possibilités d'investigation.

\section{I.2 APPROCHE DYNAMIQUE}

Cette approche est a priori la mieux adaptée lorsque la référence temporelle est indispensable : c'est le cas pour des applications de gestion fine (ex. optimisation des règles de gestion de réservoirs). Par opposition à la précédente, l'approche dynamique fournit des résultats sous forme de séries temporelles et repose sur des modèles représentant le cycle de l'eau à différents niveaux. Les prévisions des débits peuvent être issues du couplage d'un modèle climatique global ou régional et d'un modèle hydrologique. Les modèles dynamiques sont également variés mais basés essentiellement sur des séries de pluie et température ce qui contraint le choix des modélisateurs et peut faciliter les comparaisons. L'approche dynamique est encore rarement mise en œuvre [7][8][9]. En France, on citera les travaux récents portant sur le couplage entre le modèle hydrologique ISBA-MODCOU d'une part et les prévisions d'ensemble du Centre Européen pour la Prévision Météorologique à Moyen Terme d'autre part [10].

\section{II — MODÉLISATION D'UN INDICE RÉGIONAL CARACTÉRISANT LA SÉVÉRITÉ DES ÉTIAGES EN FRANCE}

L'approche présentée ici s'appuie sur une technique proposée dans le cadre du projet européen ARIDE [11] (Assessment of the Regional Impact of Droughts in Europe, ENV-CT97-0553) axé sur l'étude des sécheresses. L'absence de partenaire français et le manque de données hydrométriques post 1992 ont conduit l'élimination de la France dans le jeu de données test d'ARIDE. Les analyses présentées ici ont été menées dans le cadre d'une collaboration entre le Cemagref et le CEH. Elles visent à évaluer la méthode sur les cours d'eau français. L'application s'appuie sur 121 bassins jaugés faiblement influencés (période 1965-2004) et 20 stations pluviométriques (1994-2005).

L'outil testé est un modèle statistique conditionné par la fréquence de type de temps et illustre les liens existant entre phénomènes à grande échelle et hydrologie de surface. L'objectif est d'estimer chaque jour la valeur du Regional Deficit Index $(R D I)$ caractérisant l'extension spatiale des épisodes de sécheresse hydrologique sur des régions hydrologiquement homogènes. Ce n'est donc pas un débit que l'on cherche à prévoir mais un indicateur de l'état du système. La variable s'obtient comme suit.

La première étape consiste à construire un indicateur journalier de sévérité des étiages au niveau de chaque station (Figure 1). Pour chaque station de débits, on extrait toutes les valeurs observées sur une fenêtre centrée autour du jour $j$ de toutes les années disponibles caractérisant le régime de cette période de l'année (1). On les classe pour établir des courbes de débits classés $\operatorname{CDC}(j), j=1,365$, conditionné au jème jour de l'année (2), pour en extraire les quantiles caractéristiques (3). La position relative aux quantiles indique une relative abondance/pénurie. On caractérise l'état de la rivière par la fréquence au dépassement du débit observé le jour $j$ de chaque année (4).

L'objectif annoncé étant d'identifier et de modéliser les étiages sévères, définis ici par $D I$ (Deficit Index) dépendant du seuil $Q 90$, dépassé $90 \%$ du temps. $D I(t)$ prend la valeur 1 si pour ce jour $t$ (le jème de l'année civile) le débit est inférieur à la valeur $\mathrm{Q} 90(j)$ et 0 sinon.

L'étape suivante est de constituer une variable à pertinence régionale. Des régions regroupant les cours d'eau soumis simultanément à des étiages sévères sont établies à partir d'une méthode de classification objective. Deux techniques disponibles dans les packages $R$ et baptisées $A G N E S$ et $P A M$ (http://www.r-project.org) ont été mises en oeuvre. Toutes deux proposent des régions assez similaires mais les regroupements suggérés montrent une dépendance à la période 

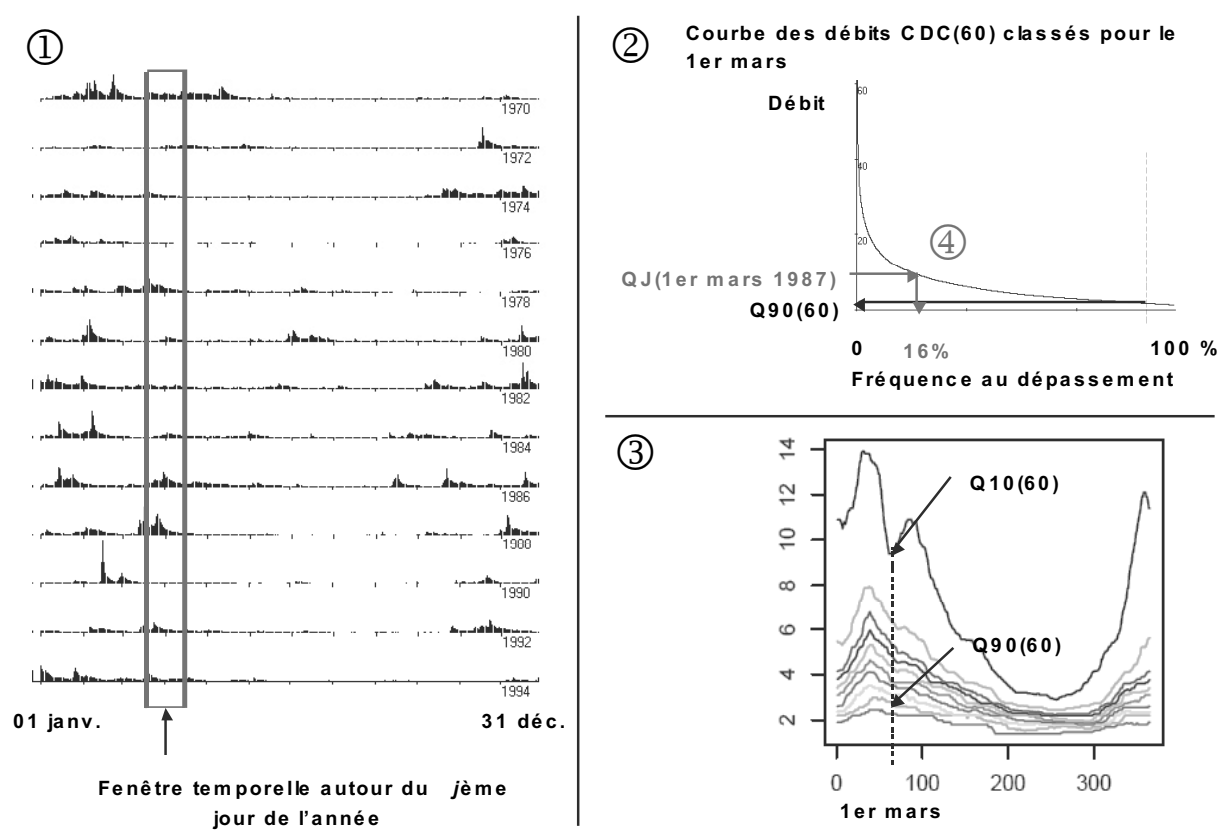

Figure 1 : Procédure de calcul de l'indice de sévérité local DI

d'étude considérée. La configuration à six régions semble la moins sensible au choix de la période de référence et à l'algorithme de classification et a été retenue ici. Finalement, les contours des groupes proposés par la procédure de partition la plus cohérente spatialement ont été modifiés pour garantir une meilleure homogénéité en termes de processus hydrologiques (Figure 2). La variable régionale $R D I$ est obtenue en moyennant les valeurs de $D I$ au sein de chaque région. $R D I$ est interprétable en tant que proportion de stations en situation d'étiages sévères :

$$
R D I(t)=\frac{1}{n} \sum_{n \text { stations } \in \text { Région }} D I(t)
$$

où $n$ est le nombre de stations de la région examinée.

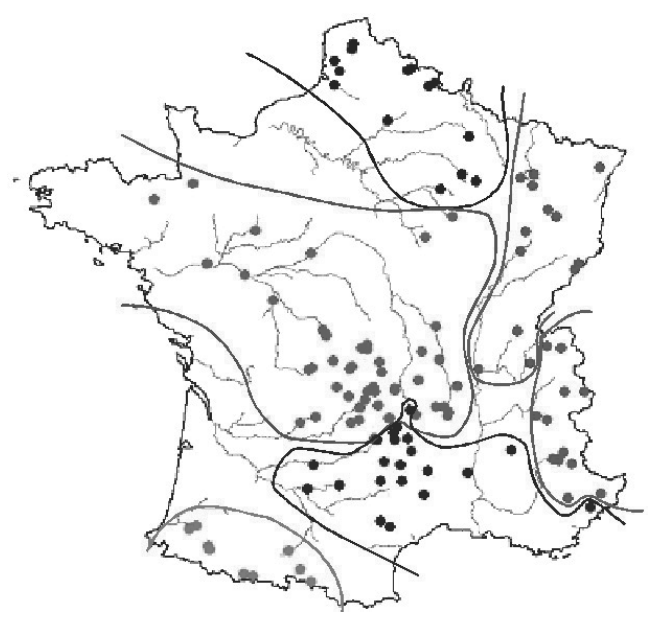

Figure 2 : Découpage de la France métropolitaine en six secteurs homogènes vis à vis des fluctuations de DI
Pour la prévision, on cherche à quantifier les évolutions de l'extension des sécheresses au niveau d'une région d'un jour à l'autre en se basant sur le type de temps du jour $j$. Il est entendu qu'une situation associée à une forte probabilité (resp. faible probabilité) de précipitations aura tendance à réduire (resp. augmenter) le nombre de stations en état de sécheresse. Notre travail s'est appuyé sur la classification européenne HBG (Hess-Brezowsky Grosswetterlaggen) [12]. L'opération suivante consiste à établir un lien entre $R D I$ (i.e. l'aggravation ou la réduction de l'état de sécheresse régionale) et l'occurrence d'une situation synoptique. Pour une manipulation plus aisée, nous avons regroupé les 29 classes initiales en un nombre réduit de groupes ayant un sens hydrologique. Nous avons construit $m$ classes selon le risque de pluie (i.e. évalué par la proportion de jours dont le cumul de pluie a dépassé $1 \mathrm{~mm}$, calculée sur l'ensemble des jours de chaque classe HBG). Ces $m$ classes contiennent chacune en moyenne $(100 / m) \%$ des situations. Finalement, les types de temps avec un risque équivalent de pluie sont placés dans le même groupe. Ce travail a été effectué pour chaque région par saison et par an en s'appuyant sur l'information issue des pluviomètres.

La variable $R D I$ étant fortement auto-corrélée, on considère le modèle suivant :

$$
R D I(t)=\frac{y(t)-y_{0}}{1-y_{0}} \text { avec } y(t)=y(t-1)+\sum_{i=1}^{m} b_{i} Z_{i}(t),(2)
$$

où $m$ est le nombre de types de temps. $Z_{i}(t)$ prend la valeur 1 si on observe le type de temps $i$ le jour $t$ et 0 sinon. Les paramètres $y_{0}$ et $b_{i}$ sont ajustés par optimisation pour chaque région sur la période 1961-2000 sous contrainte (par définition, la moyenne temporelle de $R D I$ doit être égale à 0.10 ). Des tests ont été réalisés avec $m=4$ et $m=8$. En phase 
de calage, les coefficients de corrélation $r$ entre observation et reconstitution sont de l'ordre de 0.5. Les meilleurs résultats sont obtenus pour les régions nord et ouest, où l'influence pluviale sur les régimes hydrologiques est marquée (au mieux $r=0.65$ pour $m=8$ avec un découpage saisonnier pour la région nord). Le modèle peine à reconstituer $R D I$ sur les secteurs alpins et pyrénéens.

La qualité prédictive de la méthode est mesurée sur les années 2001-2004, période réservée pour la validation. Le modèle est donc initialisé le $1^{\text {er }}$ janvier 2001 et non réinitialisé en cours de modélisation. Les résultats sont très contrastés : les coefficients de corrélation peuvent chuter fortement ( $r=0.05$ contre 0.43 en calage pour $m=8$ avec un découpage saisonnier pour la région pyrénéenne). La sécheresse de l'année 2003 est en partie bien reconstituée sur l'ensemble des régions (exemples en Figure 3). Dans l'état actuel, sous scénario futur parfaitement connu, les résultats montrent que le modèle ne serait pas très fiable en mode opérationnel. On peut néanmoins noter que sans information de pluie explicite, une classification en type de temps peut expliquer plus de $25 \%$ de la variabilité du signal débit sur certaines régions.
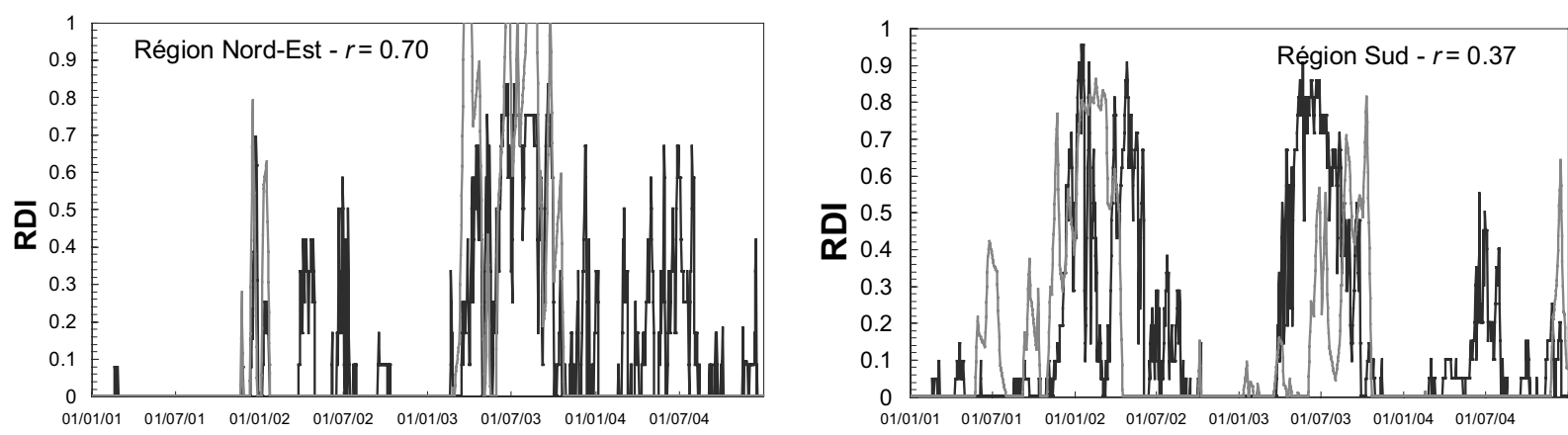

Figure 3 : Reconstitution du RDI sur la période de contrôle 2001-2004 sur deux régions (en noir : observations ; en gris : simulations avec une classification saisonnalisée comportant huit groupes)

\section{USAGE D'UNE MODÉLISATION PLUIE DÉBIT CONDITIONNÉE PAR DES SCÉNARIOS HYDRO- MÉTÉOROLOGIQUES FUTURS}

Dans le cadre du projet européen NeWater (www.newater. org), le Cemagref a exploré la faisabilité d'outils de prévision des débits à moyen terme sur le bassin du Rhin. Un travail de modélisation hydrologique a été engagé à l'aide de données journalières pluie/débit fournies par RIZA (http://www. rijkswaterstaat.nl), institut chargé de la veille hydrologique au Pays-Bas. Les objectifs de ce travail étaient les suivants :

proposer une solution compacte pour la prévision à moyen terme sur les sept sous-bassins du Rhin, en alternative à une modélisation lourde déjà existante sur le bassin [13]

explorer les possibilités de prévision à 30,60 et 90 jours.

Les données disponibles pour ce travail comportaient des séries de débits journaliers sur la période 1970-1995 pour les sept stations hydrométriques de mentionnées sur la Figure 4 ainsi que des données de pluie journalières sur 134 postes répartis sur l'ensemble du bassin du Rhin.

\section{III.1 QUELS MODÈLES POUR UNE APPROCHE DYNAMIQUE DE LA PRÉVISION D'ÉTIAGE À MOYEN TERME?}

Pour répondre aux attentes de RIZA, les choix suivants ont été effectués :
- mise en œuvre d'une approche mixte dynamique/statistique (Figure 5) : dans un premier temps, un modèle de transformation pluie/débit dynamique est mis au point sur chacun des bassins cibles. Ces modèles sont ensuite alimentés par des scénarios de pluie générés à l'aide d'un outil statistique. Cette dernière option a été préférée aux sorties de modèles climatiques dans un souci d'allègement de la démarche. Des données telles que généré par le Centre Européen pour la Prévision Météorologique à Moyen Terme (http://www. ecmwf.int) sont effet très volumineuses et non-accessibles au grand public. La production de prévisions à pas de temps journalier sur un grand nombre d'années aurait donc posé problème ;

- utilisation d'un modèle de transformation pluie-débit global : dans ce type de modèle, les forçages climatiques sont considérés comme homogènes sur l'ensemble du bassin versant. L'avantage réside dans la rapidité de mise en œuvre et la parcimonie des données nécessaires pour générer des chroniques de débit. Le modèle GR4J à quatre paramètres a été utilisé ici [14]. Il utilise en entrée des séries de pluie et d'évapotranspiration potentielle moyennées sur le bassin et calcule des débits à l'exutoire. Les paramètres sont calés par une procédure automatique tentant de rapprocher au mieux les débits simulés des observations ;

- afin de tenir compte des influences neigeuses sur la Suisse, un module neige provenant du modèle suédois HBV [15] a été adjoint à GR4J. Il utilise la température comme entrée additionnelle et calcule les variations de stock neigeux ainsi que son impact sur l'écoulement. L'assemblage de GR4J et 


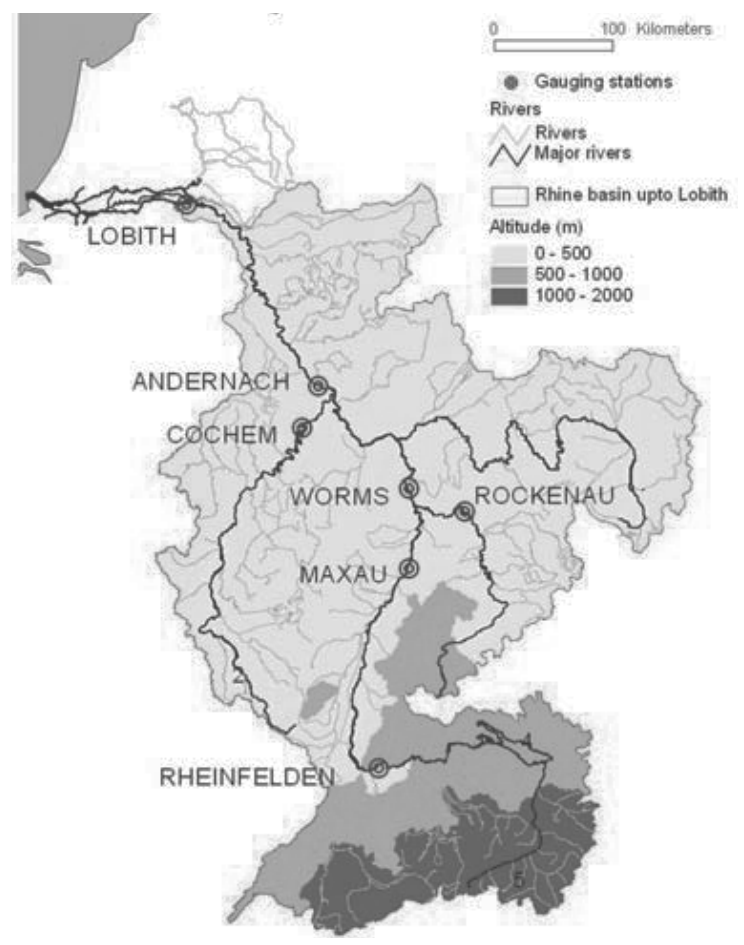

\begin{tabular}{|c|c|c|c|c|}
\hline Station & $\begin{array}{l}\text { Surf. } \\
\left(\mathrm{km}^{2}\right)\end{array}$ & $\begin{array}{c}\text { Ecoul. } \\
\text { moyen } \\
\text { annuel } \\
1970-1995 \\
(\mathrm{~mm})\end{array}$ & $\begin{array}{c}\text { Pluie } \\
\text { moyenne } \\
\text { annuelle } \\
\text { 1970-1995 } \\
(\mathrm{mm})\end{array}$ & $\begin{array}{l}\text { Période } \\
\text { d'étiage }\end{array}$ \\
\hline Rheinfelden (Rhin) & 34450 & 957 & 1321 & $\begin{array}{l}\text { décembre à } \\
\text { février }\end{array}$ \\
\hline Maxau (Rhin) & 53450 & 808 & 1510 & $\begin{array}{c}\text { décembre à } \\
\text { février }\end{array}$ \\
\hline Rockenau (Neckar) & 13230 & 333 & 1186 & août à octobre \\
\hline Worms (Rhin) & 69100 & 674 & 1317 & $\begin{array}{c}\text { décembre à } \\
\text { février }\end{array}$ \\
\hline Cochem (Moselle) & 28230 & 957 & 1321 & août à octobre \\
\hline Andernach (Rhin) & 141340 & 478 & 1069 & $\begin{array}{l}\text { septembre à } \\
\text { novembre }\end{array}$ \\
\hline Lobith (Rhin) & 160800 & 447 & 1045 & $\begin{array}{l}\text { septembre à } \\
\text { novembre }\end{array}$ \\
\hline
\end{tabular}

Figure 4 : Les sept sous-bassins du Rhin sur lesquels portent les prévisions d'étiage

du module neige de HBV forme le modèle hydrologique appliqué sur les sept bassins. On notera la bonne qualité des simulations produites par ce modèle comme indiqué dans le Tableau1 (qualité mesurée par le critère de Nash-Sutcliffe [16], $100 \%$ représente la perfection, $0 \%$ correspond à un modèle équivalent au modèle naïf donnant un débit constant égal au module). Ces performances sont notables compte tenu de la taille des bassins et de la compacité du modèle ; - comme indiqué sur la Figure 5, le modèle hydrologique est ensuite alimenté par cent scénarios de pluie produits par le générateur aléatoire KNN [17]. Ce générateur est basé sur le rééchantillonnage par voisins proches : chaque jour simulé $j$, le générateur identifie une liste de jours dans la base de donnée pluviométrique. La sélection est fondée sur des critères de similitude avec $j$ : par exemple un jour est dit « voisin » si le cumul journalier sur $n$ postes représentatifs est très proche pour les deux jours. Un voisin $k$ est ensuite choisi aléatoirement dans cette liste et la pluie de $k+1$ est affectée à $j+1$. KNN permet donc de générer simplement un grand nombre de scénarios de pluie future cohérent avec la climatologie régionale. Ces scénarios n'ont en revanche pas de caractère prédictif comme pourraient l'avoir des sorties de modèle météorologique.

Le système mis en place permet donc de produire chaque jour de la période ciblée cent hydrogrammes prévus couvrant les quatre-vingt-dix jours suivants. Les périodes d'étiage sont mentionnées dans le tableau de la Figure 4. Le système est évalué rétroactivement en calant les paramètres sur la période 1970-1981 puis en lançant des prévisions sur la période 1982-1995.

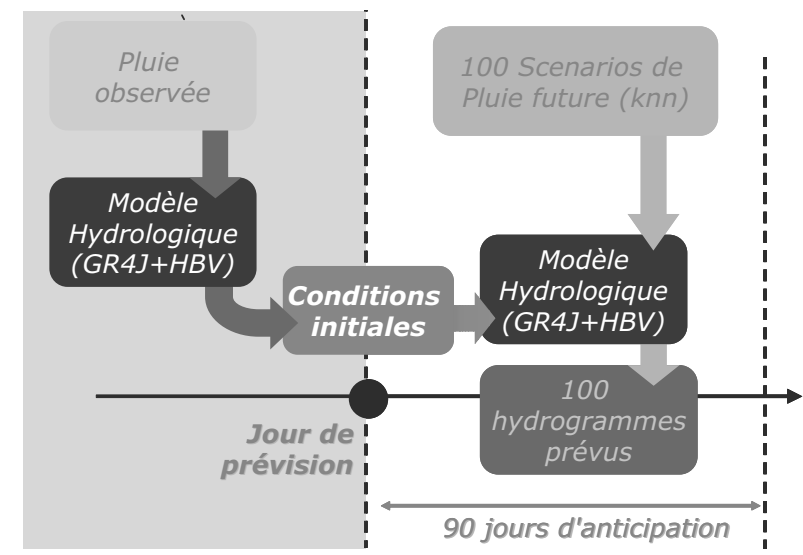

Figure 5 : Système de prévision mis en ouvre pour réaliser les prévisions d'étiage sur le bassin du Rhin

\section{III.2 COMMENT ÉVALUER LA QUALITÉ D'UNE PRÉVISION PROBABILISTE EN HYDROLOGIE?}

Le développement d'outils de simulation numérique doit impérativement s'accompagner d'une analyse fine des résultats en comparant les sorties du modèle aux observations. La comparaison entre modèles, pour être objective, doit être effectuée à l'aide de critères numériques qui placent chaque structure sur un pied d'égalité et distinguent les meilleures. 
La visualisation graphique, toujours nécessaire, n'est pas écartée mais elle intervient dans un deuxième temps.

Dans le cas présent, les critères classiques utilisés en prévision de crue et simulation (Nash-Sutcliffe et critère de persistance) ne sont adaptés pour deux raisons :

- ils sont adaptés à un cadre déterministe dans lequel le modèle ne produit qu'un seul calcul à chaque pas de temps. Ici, le cadre de travail est probabiliste avec cent hydrogrammes produits par le modèle pour chaque jour de prévision ;

- ils ne mesurent que l'écart entre calcul et observation par le biais de l'erreur quadratique moyenne. En prévision probabiliste, la dispersion des prévisions constitue un second élément à mesurer.

Dans la continuité des travaux réalisés par les météorologistes [1][10][18][19], deux critères ont été proposés pour quantifier la qualité des prévisions. Pour ces deux indicateurs, la valeur 1 correspond à une prévision parfaite et 0 à une prévision équivalente au modèle de référence.

Le critère $C R 1$ mesure le biais entre observé et prévu. Il est défini comme l'écart entre les débits observés et la moyenne des 100 prévisions, rapporté à l'écart entre les débits observés et le meilleur de deux modèles de références : la persistance (prévision égale au dernier débit observé) et le débit moyen interannuel pour chaque jour julien :

$$
\operatorname{CR} 1(L)=\frac{1-\sum_{i=1}^{N b J \text { prev }}\left(\log \left[\overline{P_{i}}(L)\right]-\log \left[O_{i}\right]\right)^{2}}{\min \left\{\sum_{i=1}^{N b J \text { prev }}\left(\log \left[O_{i-L}\right]-\log \left[O_{i}\right]\right)^{2}, \sum_{i=1}^{N b J \text { Jrev }}\left(\log \left[O_{i}^{\text {IntA }}\right]-\log \left[O_{i}\right]\right)^{2}\right\}}
$$

où $L$ désigne le délai de prévision en jour, NbJprev le nombre de jours de prévision, $O_{i}$ le débit observé le jour $i\left(\mathrm{~m}^{3} / \mathrm{s}\right)$, $O_{i}^{\text {IntA }}$ le débit moyen interannuel du jour $i\left(\mathrm{~m}^{3} / \mathrm{s}\right)$ et $P_{i}\left({ }_{\mathrm{L}}\right)$ la moyenne des 100 débits prévus pour le jour $i$ depuis le jour $i-L\left(\mathrm{~m}^{3} / \mathrm{s}\right)$. Dans la formule (3), la transformation logarithmique permet de mettre l'accent sur les faibles débits.

Le second critère mesure la dispersion des prévisions rapportée à la dispersion interannuelle des débits observés. Sur la base du critère de Brier [1], cette dispersion est évaluée en comparant des fréquences d'apparition observées et prévues. Ces fréquences sont calculées pour quatre classes de débit en privilégiant les faibles valeurs : débit inférieur au quantile $10 \%$, compris entre 10 et $25 \%$, entre 25 et $50 \%$ et supérieur à $50 \%$. Ceci permet de calculer le critère $C R 2$ suivant, baptisé critère d'incertitude :

$$
C R 2(L)=\frac{1-\sum_{c l=1}^{4} \sum_{i=1}^{N b J p r e v}\left(\hat{F}_{c l, i}(L)-F_{c l, i}\right)^{2}}{\sum_{c l=1}^{4} \sum_{i=1}^{N b J p r e v}\left(F_{c l, i}^{\text {Int.Ann }}-F_{c l, i}\right)^{2}}
$$

où $F_{c l, i}$ désigne l'appartenance du débit observé le jour $i$ à la classe $c l$ (vaut 0 ou 1), $\hat{F}_{c l, i}(L)$ le pourcentage des débits prévus pour le jour $i$ appartenant à la classe $c l$ (varie entre 0 et 1) et $F_{c l, i}^{\text {Int.Ann }}$ la fréquence d'apparition interannuelle du débit dans la classe $c l$ pour le jour $i$ (varie entre 0 et 1 ).

\section{III.3 ANALYSE DES RÉSULTATS}

La Figure 6 montre l'évolution des deux critères pour trois stations et des prévisions avec un horizon de prévision allant de 1 à 90 jours. La Figure 6 et le Tableaul permettent de retenir les points suivants concernant la prévision d'étiage sur le bassin du Rhin :

— en termes de biais : au-delà de 30 jours, la méthode adoptée ici ne permet pas de fournir des prévisions significativement meilleures que la persistance ou le débit interannuel du jour (critère $C R 1$ proche de 0 sur la Figure 6). Ce délai s'avère même plus court sur l'amont du bassin. Ceci pourrait être dû au caractère très réactif des bassins alpins ; - en termes d'incertitude : le délai est encore plus court voire inexistant pour la partie du bassin sous influence neigeuse (CR2 est négatif ou nul sur la Figure 6). C'est une conclusion importante de ce travail : une prévision peut être performante du point de vue du biais, i.e. la moyenne des prévisions est informative, mais pauvre vis à vis des incertitudes, i.e. avec une grande dispersion.

\section{CONCLUSION}

Les deux applications montrent les progrès à réaliser avant d'obtenir des prévisions saisonnières. Le premier modèle appliqué est relativement naïf. Il exploite une information plus sophistiquée que celle issue des grands indicateurs (par ex. NAO ou SST) mais moins détaillée que celle fournie par les données pluviométriques. L'application réalisée en France montre qu'une description même sommaire de la situation synoptique intégrée dans un modèle linéaire très simple (réaction quasi immédiate du système aux impulsions de pluie, non prise en compte des stocks) peut expliquer jusqu'à $40 \%$ de la variabilité de l'indicateur de sévérité d'étiage en phase de calage. Ce résultat n'était pas acquis d'avance. Les qualités prédictives du modèle auraient pu être étudiées plus en détail en simulant stochastiquement des chroniques possibles de situations météorologies déclinées en type de temps. Cependant, d'une part, les résultats n'auraient pas pu être meilleurs que ceux obtenus en futur parfaitement connu ; d'autre part, comment conditionner les scénarios futurs? Ce point rejoint les faiblesses du système mis en œuvre sur le Rhin. La prévision reste fiable les premiers jours, lorsque que la mémoire du bassin gouverne l'évolution des débits. $\mathrm{Au}$ delà, ce sont les entrées météorologiques qui vont principalement faire évoluer les débits. La question principale et encore ouverte est d'identifier des prédicteurs descriptifs de l'état de l'atmosphère ou de l'océan au voisinage de la France facilement accessibles et surtout efficaces pour piloter un modèle statistique (du type II) ou conditionner un générateur stochastique (du type III). Il restera également à comparer les résultats avec le même modèle alimentés par 
Tableau 1 : Résultats obtenus en simulation (modèle GR $4 J+H B V$ ) et prévision (modèle GR $4 J+H B V+$ simulateur KNN)

\begin{tabular}{|c|c|c|c|c|c|c|}
\hline \multirow[b]{3}{*}{ Station } & \multirow{2}{*}{\multicolumn{2}{|c|}{$\begin{array}{c}\begin{array}{c}\text { Critère sur la simulation } \\
\text { (Nash-Sutcliffe en contrôle) }\end{array} \\
1982-1995\end{array}$}} & \multirow{2}{*}{\multicolumn{2}{|c|}{$\begin{array}{c}\text { Critères sur la prévision } \\
\text { à } 30 \text { jours } \\
1982-1995\end{array}$}} & \multirow{2}{*}{\multicolumn{2}{|c|}{$\begin{array}{c}\text { Critères sur la prévision } \\
\text { à } 60 \text { jours } \\
1982-1995\end{array}$}} \\
\hline & & & & & & \\
\hline & $\mathrm{Q}$ & $\log (\mathrm{Q})$ & CR1 & $\mathrm{CR} 2$ & CR1 & $\mathrm{CR} 2$ \\
\hline Rheinfelden (Rhin) & 81.6 & 84.7 & $11 \%$ & $-6 \%$ & $-20 \%$ & $-14 \%$ \\
\hline Maxau (Rhin) & 81.9 & 84.7 & $11 \%$ & $-4 \%$ & $-4 \%$ & $-9 \%$ \\
\hline Rockenau (Neckar) & 82.7 & 89.3 & $1 \%$ & $-6 \%$ & $-8 \%$ & $-11 \%$ \\
\hline Worms (Rhin) & 86.9 & 84.0 & $11 \%$ & $-4 \%$ & $2 \%$ & $-9 \%$ \\
\hline Cochem (Moselle) & 87.6 & 91.4 & $4 \%$ & $-6 \%$ & $-35 \%$ & $-10 \%$ \\
\hline Andernach (Rhin) & 89.2 & 88.2 & $21 \%$ & $-2 \%$ & $2 \%$ & $-4 \%$ \\
\hline Lobith (Rhin) & 87.2 & 85.1 & $18 \%$ & $-2 \%$ & $0 \%$ & $-5 \%$ \\
\hline
\end{tabular}
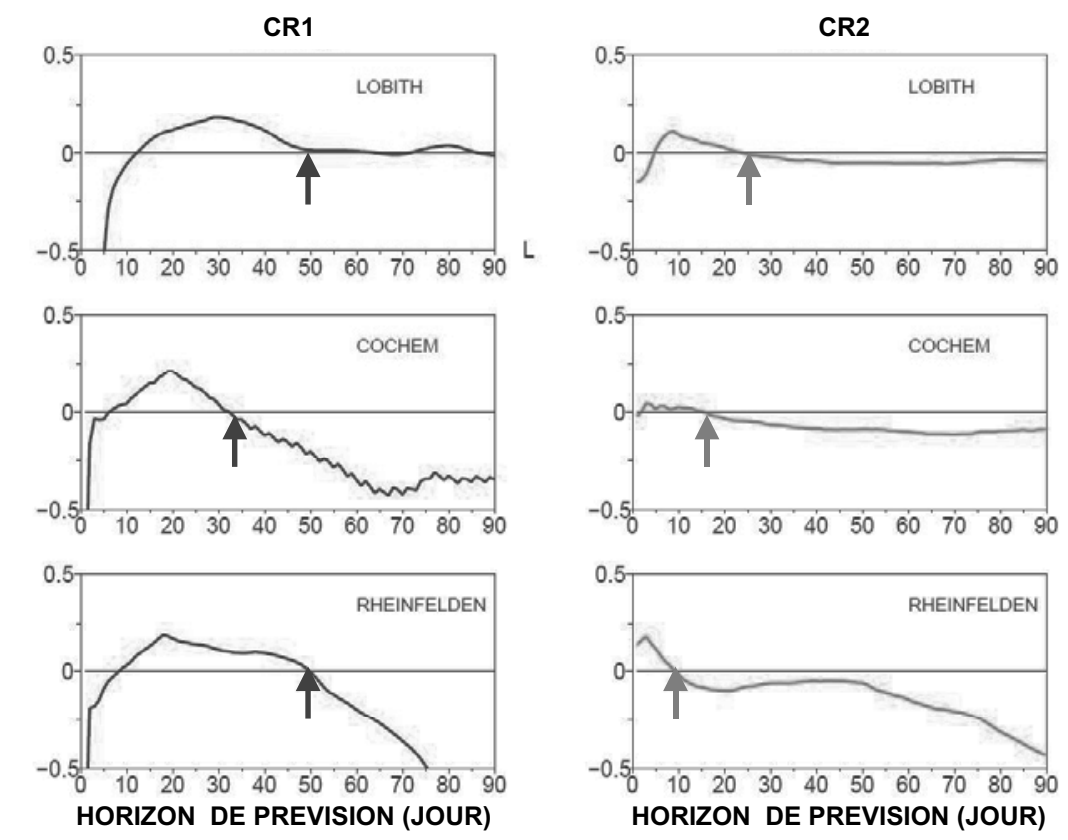

Figure 6 : Valeurs des critères $C R 1$ et $C R 2$ d'évaluation des prévisions d'étiage en trois stations (Lobith, Cochem, Rheinfelden)

des scénarios issus d'un modèle météorologique et enfin autre difficulté, d'inclure les actions humaines.

\section{V — RÉFÉRENCES}

[1] BRIER G. W. (1950) - Verification of forecasts expressed in terms of probability. Monthly Weather Review. 78(1) 1-3
[2] PIEChOTA T. C., DRACUP J. A. (1999) - Long-range streamflow forecasting using El-Nino Southern Oscillation indicators. Journal of Hydrologic Engineering. 4(2) 144-151

[3] SOUZA FILHO F. A., LALL U. (2003) - Seasonal to interannual ensemble streamflow forecasts for Ceara, Brazil : Applications of a multivariate, semiparametric algorithm. Water Resources Research, doi 10.1029/2002WR001373. 39(11) 1307

[4] ARAGHINEJAD S., BURN D. H., AND KARAMOUZ M. (2006) — Long-lead probabilistic forecasting of streamflow 
using ocean-atmospheric and hydrological predictors. Water Resources Research, doi 10.1029/2004WR003853. 42, W03431

[5] SVENSSON C., PRUDHOMME C. (2005) — Prediction of British summer river flows using winter predictors. Theoretical Appl. Climatol. 82 1-15

[6] WILBY R. L., WEDGBROW C. S., FOX H. R. (2004) Seasonal predictability of the summer hydrometeorology of the River Thames, UK. Journal of Hydrology. 295 1-16

[7] HAMLET A. F., LETTENMAIER D. P. (1999) - Columbia river streamflow forecasting based on ENSO and PDO climate signals. Journal of Water Resources Planning and Management. 125(6) 333-341

[8] FRANZ K. J., HARTMANN H. C., SOROOSHIAN S., BALES R. (2003) - Verification of National Weather Service Ensemble Streamflow Predictions for water supply forecasting in the Colorado River Basin. Journal of Hydrometeorology. 4(6) $1105-1118$

[9] TUCCI C., CLARKE R. T., COLLISCHONN W., DA SILVA DIAS P. L., DE OLIVEIRA G. S. (2003) - Longterm flow forecasts based on climate and hydrologic modeling : Uruguay River basin. Water Resources Research, doi 10.1029/2003WR002074. 39(7) 1181

[10] ROUSSET-REGIMBEAU F. (2007) - Modélisation des bilans de surface et des débits sur la France, application à la prévision d'ensemble des débits. Thèse, Université Toulouse III.

[11] DEMUTH S., STALH K. (2001) - ARIDE - Assessment of the Regional Impact of Droughts in Europe. Institute of Hydrology, University of Freiburg. 154 pages
[12] HESS P., BREZOWSKI H. (1977) - Katalog der Grobwetterlagen Europas 1881-1976. 3 verbesserte und ergäntze Auflage. Berichte des Deutschen Wetterdienstes. 113

[13] INSTITUTE FOR INLAND WATER MANAGEMENT AND WASTE WATER TREATMENT AND FEDERAL INSTITUTE OF HYDROLOGY (2005) - Daily HBV Model for the Rhine Basin. Hydrological Modelling in the River Rhine Basin. Part III 227 pages

[14] PERRIN C., MICHEL C., ANDREASSIAN V. (2003) Improvement of a parsimonious model for streamflow simulation. Journal of Hydrology. 279(1-4) 275-289

[15] BERGSTRÖM S. (1996) - The HBV model. Computer models of watershed hydrology, V.P. Singh (Editor), Water Resources Publications, Highlands Ranch, CO 1995.

[16] NASH J. E., SUTCLIFFE J. V. (1970) - River flow forecasting through conceptual models. Part 1 : a discussion of principles. Journal of Hydrology. 10(3) 282-290

[17] LALL U., SHARMA A. (1996) — A nearest neighbour bootstrap for resampling hydrologic time series. Water Resources Research. 32(3) 679-693

[18] WILKS D. S. (2000) - On Interpretation of Probabilistic Climate Forecasts. Journal of Climate. 13(11) 1965-1971

[19] ROULIN E., VANNITSEM S. (2005) - Skill of mediumrange hydrological ensemble predictions. Journal of Hydrometeorology. 6(5) 729-744 